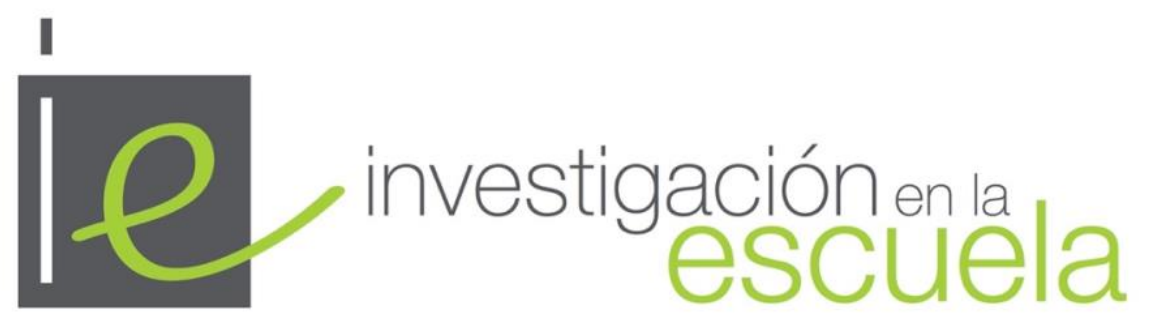

Revista de Investigación e Innovación Educativa n ${ }^{\circ}$ X, 2020 | e-ISSN 2443-9991

\title{
Emociones en tareas de escritura comunitaria en Educación Secundaria
}

\author{
Emotions in community writing tasks in Secondary Education
}

Rebeca Mariel Martinenco es Becaria Doctoral del CONICET en el Instituto Académico Pedagógico de la
iD Universidad Nacional de Villa María (Argentina) · rebecamartinenco@gmail.com · https://orcid.org/0000-00022781-3381

Rocío Belén Martín es Investigadora Asistente del CONICET en el CIT Villa María y Profesora Adjunta en la Facultad de Ciencias Exactas, Físicas y Naturales de la Universidad Nacional de Córdoba (Argentina) rociobelenmartin@gmail.com/rbmartin@unc.edu.ar·https://orcid.org/0000-0003-3172-0070

Leticia García Romano es Investigadora Asistente del CONICET y Profesora Titular en la Facultad de Ciencias

iD Exactas, Físicas y Naturales de la Universidad Nacional de Córdoba (Argentina) · leticia.garcia@unc.edu.ar https://orcid.org/0000-0003-3552-0287

Cómo citar este artículo

Martinenco, R.M., Martín R. B. y García Romano, L. (2020). Emociones en tareas de escritura comunitaria en Educación Secundaria. Investigación en la Escuela, 102, 97-108. doi: http://doi.org/10.12795/IE.2020.1102.07

Resumen. La dinámica emocional en los aprendizajes y las comunidades son temas recurrentes en la bibliografía actual disponible. Sin embargo aún son más limitados los estudios que, desde una metodología de diseño, pretenden promover emociones positivas en los estudiantes. De allí deriva el propósito del presente escrito, el cual radica en comprender las emociones experimentadas por un grupo de estudiantes de sexto año de Educación Secundaria frente a tareas de escritura comunitaria que fueron diseñadas para fomentar el despliegue de emociones positivas, reconociendo que éstas son dinámicas y fluctúan en diferentes momentos. Para alcanzar este objetivo, el escrito se estructura de la siguiente manera. En primer lugar, se brindan contextualizaciones teóricas acerca de las emociones y las comunidades de escritores. Luego, el apartado Material y Métodos recoge las principales características de la metodología de diseño, además de especificaciones referidas a los participantes del estudio, las estrategias de recolección de datos empleadas y los procedimientos desarrollados. Los resultados de la investigación destacan las emociones experimentadas frente a la escritura libre, la escritura grupal y la escritura con TIC. A modo de discusión, se plantea un énfasis en las emociones positivas experimentadas por los estudiantes respecto a escrituras grupales y autorreguladas por ellos mismos; también se destacan las limitaciones encontradas y las potenciales líneas de investigación a futuro.

Abstract. Emotional dynamics in learning and communities are recurring themes in the current available literature. However, the studies that, from a design methodology, seek to promote positive emotions in students are even more limited. Hence the purpose of this writing, which is to understand the emotions experienced by a group of sixth-year students of Secondary Education in front of community writing tasks that were designed to encourage the display of positive emotions, recognizing that these are dynamic and fluctuate in different moments. To achieve the main goal, the writing is structured as follows. First, theoretical contextualizations about emotions and communities of writers are provided. Then, the Material and Methods section collects the main characteristics of the design methodology, as well as specifications regarding the study participants, the data collection strategies used and the procedures developed. The research results highlight the emotions experienced in front of free writing, group writing and writing with ICT. As a discussion, an emphasis is put on the positive emotions experienced by students regarding group and selfregulated writing. The limitations found and the potential lines of future research are also highlighted. 


\section{Palabras clave $\cdot$ Keywords}

Emoción, aprendizaje en grupo, secundaria segundo ciclo, actividades escolares, escritura, clase. Emotion, group learning, upper secondary, school activities, writing, class.

\section{Introducción y estado de la cuestión}

Desde hace algunos años, diferentes perspectivas provenientes de la Psicología Educacional vienen advirtiendo acerca de la importancia que poseen las emociones en la práctica educativa (Fernández-Berrocal et al., 2017; Järvenoja y Järvelä, 2009; Pekrun, 2006).

Actualmente en investigación es recurrente el interrogante acerca de las actividades que se llevan a cabo dentro del aula para mejorar la educación emocional del alumnado. De aquí surge el interés por el estudio de los procesos emocionales en los estudiantes de Educación Secundaria empleando una metodología de diseño. El propósito de este trabajo radica en comprender las emociones experimentadas por un grupo de estudiantes frente a tareas de escritura comunitaria, considerando fundamentalmente la dinámica emocional y los modos de participación que se despliegan en este proceso. En este estudio se propusieron tareas de aprendizaje para promover emociones positivas en los estudiantes, reconociendo que éstas son dinámicas, cambiantes y van modificándose en diferentes momentos.

El escrito se organiza de la siguiente manera: en primer lugar, se hace referencia al concepto de emoción en general y vinculado a los ambientes educativos, en particular; también se destaca la potencialidad de la comunidad de escritores y la importancia que adquiere la participación al interior de las mismas. En segundo lugar, se precisan los materiales y métodos que guían el estudio. En tercer lugar, se presentan el análisis y los resultados de la investigación. En cuarto lugar, se exponen discusiones acerca de los aportes derivados del escrito.

\subsection{Emociones}

Desde hace unos años, ciertos autores reconocen la importancia que posee el estudio de las emociones de los estudiantes. Goetz et al. (2006) especifican cuatro motivos por los que resulta interesante investigar esta temática: en primer lugar, porque las experiencias emocionales de los estudiantes están vinculadas directamente a su bienestar subjetivo; en segundo lugar, puesto que las emociones inciden en la calidad de los aprendizajes y los logros de los estudiantes; en tercer lugar, dado que éstas influyen en la comunicación en el aula y, por lo tanto, en la interacción con los docentes y el resto de los estudiantes; finalmente, porque estos estudios posibilitan el diseño de intervenciones que fomenten las emociones, el aprendizaje y los logros.

Además, las emociones han adquirido preponderancia al interior de las instituciones educativas, en parte por la intención de brindar una formación integral, dentro de la cual es central la dimensión emocional (Bisquerra, 2018).

Tal vez por ello encontramos numerosos estudios acerca de emociones que despliegan los estudiantes en los diferentes niveles de educación. Se hallaron investigaciones llevadas a cabo en instituciones educativas de educación infantil, como la realizada por Putwain et al. (2020) en clases de Matemática, en la que hallaron influencia del control de las emociones sobre resultados de aprendizaje positivos. En Educación Secundaria, es posible mencionar estudios sobre cómo el aburrimiento y el disfrute en clases de Lengua española inciden sobre aspectos motivacionales y de rendimiento de los estudiantes (González et al., 2013); también se encuentran investigaciones en clases de ciencias experimentales, tal como la desarrollada por Vázquez-Bernal et al. (2020), quienes hallaron predominio de emociones positivas antes, durante y después de la visita a un elemento patrimonial -Molino de mareas Zaporito-. En Educación Universitaria, destacan las investigaciones realizadas con docentes en formación. Tal es el caso de un estudio con futuros profesores de Educación Primaria, donde se halló una vinculación significativa entre el recuerdo de las emociones experimentadas al aprender Biología en Educación Secundaria y el aprendizaje de estos conceptos construidos en ese momento (Ochoa de Alda et al., 2019).

Tal como se puede vislumbrar, también fueron diversas las áreas en las que se desarrollaron estudios sobre las emociones. Particularmente en España son destacables los estudios en el área de Ciencias que subrayan la importancia de abordar las emociones a fin de planificar los procesos de enseñanza y aprendizaje de modo que resulten óptimos para los estudiantes (Dávila Acedo et al., 2016; Mellado et al., 2014).

$\mathrm{El}$ auge del estudio de las emociones reveló una recurrente falta de acuerdo en el intento de definirlas. En términos de Chóliz (2005), esto se debe a que las emociones son multidimensionales y, en parte, las diferencias en su conceptualización residen en el papel que cada modelo teórico otorga a las diferentes dimensiones. En este sentido, Reeve (2010) postula que las emociones involucran cuatro componentes: 
sentimental, biológico, intencional y social-expresivo. De modo similar, Bisquerra (2016a) advierte que en la respuesta emocional se distinguen los aspectos psicofisiológico, comportamental y cognitivo y, aportando a esta complejidad de la emoción, Mellado et al. (2014) brindan una definición que involucra estas diversas dimensiones al advertir en este concepto componentes biológicos y subjetivos que se vinculan al ambiente y presentan influencia individual y social.

De esta manera es posible comprender lo complejo que resulta el estudio de las emociones y más aún en ámbitos educativos, donde diversos factores se ponen en juego. La Teoría del control-valor propuesta por Pekrun es un intento por comprender de modo integral las emociones vinculadas a los aprendizajes y a los logros que se despliegan en los ambientes escolares (Martinenco et al., 2020; Pekrun, 2006). Según esta teoría, los estudiantes experimentan cuatro tipos de emociones en relación a sus aprendizajes. Un tipo son las emociones de logro, aquellas asociadas a los resultados obtenidos al realizar una tarea como orgullo por una resolución exitosa o vergüenza frente a un fallo. También existen las emociones epistémicas, es decir, las que se desencadenan de un problema cognitivo como la sorpresa ante a una nueva tarea o la frustración frente a los obstáculos. Un tercer tipo de emociones que los estudiantes pueden experimentar son aquellas que se refieren específicamente a los temas desarrollados en las clases; tal es el caso de la empatía experimentada al leer las peripecias de un personaje en una novela. Finalmente se destacan las emociones sociales como amor, compasión, ira o envidia; éstas son desplegadas por los estudiantes en la interacción con sus docentes y compañeros (Pekrun, 2014).

A partir de estos postulados de la Teoría de control-valor es posible comprender la multiplicidad de emociones que experimentan los estudiantes en relación a sus aprendizajes. En un sentido similar, Bisquerra (2016b) destaca que en los ambientes educativos, además de las emociones consideradas básicas -miedo, ira, tristeza, alegría, sorpresa y asco-, otras cuatro emociones poseen un papel central en la educación: en primer lugar, las emociones sociales que experimentamos en la relación con los demás como la vergüenza, la culpabilidad, el pudor, entre otras; en segundo lugar, las emociones estéticas que surgen ante las obras de arte y la belleza; en tercer lugar, el amor y en cuarto lugar, la felicidad. Estas últimas dos son consideradas por el autor como emociones positivas no básicas pero necesarias para el bienestar personal y social. Sin embargo, dicha clasificación no agota la variedad y diversidad de emociones existentes y los modos de nombrarlas. Por esta razón, el autor propone el término universo de emociones entendido como una representación de familias de emociones. Así, por ejemplo, la familia de miedo incluye temor, horror, pánico, terror, pavor, desasosiego, susto, fobia, entre otras.

En Argentina, desde los lineamientos curriculares de la provincia de Córdoba, se apuesta por una educación y formación integrales que promuevan el desarrollo de las dimensiones cognitiva, ética, afectiva, social y política. Los aprendizajes emocionales permiten a los estudiantes percibir, reconocer y comprender sus propias emociones a la vez que interpretar e interactuar con las emociones de los demás. Desde estos documentos se destaca también que el tipo y organización de tareas en que participan los estudiantes incide en las actitudes manifestadas frente a los saberes específicos y a la concepción de su aprendizaje, por lo que se propone crear propuestas de enseñanza que posibiliten el desarrollo de habilidades cognitivas, intrapersonales e interpersonales a partir de diversas tareas y escenarios escolares. Estas orientaciones también sostienen la importancia de estimular en los estudiantes el desarrollo de ciertas capacidades fundamentales; una de ellas se vincula al trabajo en colaboración (Gobierno de la Provincia de Córdoba, 2018). Respecto a este punto nos extenderemos en el próximo apartado.

\subsection{Comunidad de escritores}

En la actualidad es recurrente la referencia al trabajo colaborativo, donde la participación de todos los integrantes cobra central relevancia porque ayuda a comprender la construcción de conocimiento o el desarrollo de procesos creativos en comunidades de aprendizaje.

Wenger (2010) entiende que las comunidades están constituidas por diversas personas que interactúan y aprenden colaborativamente, lo que involucra nuevas concepciones acerca del conocimiento y el aprendizaje.

Algunos autores (Díez-Palomar y Flecha-García, 2010; Rodrigues de Mello, 2011) consideran que las comunidades constituyen una opción prometedora para potenciar los ambientes educativos a partir de una visión sistémica de la educación, de modo que promuevan aprendizajes más equitativos, dialógicos y democráticos. Partiendo de una revisión de la bibliografía existente, Martín (2019) recupera diversos rasgos, dimensiones y características que considera definitorias de las comunidades: metas compartidas, problemas genuinos, identidad, autonomía, feedback y construcción colaborativa del conocimiento. En consonancia con lo expuesto en el apartado previo, la autora advierte acerca de la importancia de considerar el rol de las emociones en la conformación de comunidades, las cuales se constituyen en base a relaciones 
interpersonales; por este motivo la dimensión afectiva es inherente a los estudios acerca de comunidades y trabajo colaborativo.

En el estudio que aquí se presenta adquieren relevancia las tareas académicas de escritura que los estudiantes realizaron de manera comunitaria. Se entiende a la escritura como una práctica sociocultural que habilita al ejercicio de la ciudadanía y la democracia (Pérez-Abril, 2004). Hand y Prain (2012) mencionan una serie de estudios realizados acerca de las estrategias que ayudan a los estudiantes a realizar escrituras de calidad; si bien dentro de estas estrategias se encuentra contar con consignas de redacción claras, realizar resúmenes, establecer metas, entre otras, interesa destacar específicamente una de ellas: la asistencia entre pares. Los autores retoman lo propuesto por Graham y Perin (2007, en Hand y Prain, 2012) para advertir acerca de la necesidad de que los estudiantes trabajen juntos en las clases.

Lo expuesto hasta aquí permite comprender cómo los procesos de participación son centrales en las comunidades de aprendizaje. La participación no se vincula solamente a comprometerse con actividades y personas, sino que se trata de incluirse activamente en las prácticas de las comunidades y de construir identidades al interior de las mismas (Wenger, 2010).

A partir de lo explicitado hasta el momento se sostiene entonces que las comunidades no sólo involucran una construcción colectiva de conocimientos sino también procesos emocionales y de participación que se despliegan en esa construcción conjunta (Martín et al., 2015).

\section{Metodología}

El estudio que se presenta constituye una investigación de diseño. El mismo involucra prácticas y herramientas participativas en contextos reales y situados (Gutiérrez y Penuel, 2014). Desde esta metodología se pretendió obtener la perspectiva de los participantes implicados pero a la vez se diseñó con la docente y se llevó a cabo una secuencia de tareas académicas, produciéndose intervenciones en el contexto.

Estos estudios de diseño se caracterizan por ser contextuales, participativos, iterativos, reflexivos, interactivos y organizados en ciclos sucesivos (Esteve-Mon et al., 2019). Siguiendo a Rinaudo y Donolo (2010), estos ciclos se organizan en tres fases: preparación del diseño, implementación y análisis retrospectivo. En la primera de ellas se definen las metas de aprendizaje y se diseñan las acciones -en este caso, se delinearon las tareas académicas realizadas en las clases, los recursos implementados, el tiempo requerido, entre otros-. La segunda etapa, implementación del diseño, consiste en llevar a cabo lo planificado en la etapa previa -en este momento del ciclo se realizaron las observaciones de clases-. Finalmente, la etapa de análisis retrospectivo incluye el análisis de los datos recogidos en las fases anteriores. De esta manera, desde el comienzo la totalidad de los participantes son parte de la investigación, incluso en la toma de decisiones.

Este tipo de investigaciones posee una meta teórica y una meta pedagógica o práctica. La primera de ellas se refiere al desarrollo de teoría sobre los procesos de aprendizaje y sobre los medios diseñados para apoyar a ese aprendizaje. En cambio, la meta pedagógica o práctica tiene que ver con la mejora de los procesos de enseñanza y de aprendizaje a partir de la generación de ciertas intervenciones que son avaladas por un campo de conocimiento disciplinar, por las teorías del aprendizaje y por las condiciones del contexto (Rinaudo y Donolo, 2010). En términos de Reinmann y Fiedler (2017), el conocimiento teórico al que se aspira en la investigación de diseño es posible generalmente a través de experiencias particulares. Por este motivo se comprende que cada secuencia diseñada en cada contexto es única; las condiciones en que ésta se desarrolla están condicionadas por las expectativas de los implicados, sus contextos y sus posibilidades (Pérez-Abril, 2004). En el caso del estudio que aquí se presenta, la meta teórica pretendió generar un avance en el ámbito de la Psicología Educacional vinculado a las emociones experimentadas en Educación Secundaria a partir de la escritura en comunidad, mientras que la meta pedagógica se orientó a mejorar las clases de Lengua y Literatura en que se desarrollaron dos grandes temáticas: literatura argentina contemporánea y texto argumentativo.

El interés por este estudio se encuadra dentro de las orientaciones curriculares para la Educación Secundaria brindadas por el Gobierno de la Provincia de Córdoba, Argentina (2018), dado que se advierte la necesidad de promover actividades de encuentro para propiciar un verdadero trabajo colectivo que habilite el logro de aprendizajes integrales. Se destaca también la importancia que posee el despliegue de habilidades intrapersonales e interpersonales para construir acuerdos, para valorar la tarea compartida y para reconocer lo necesario que es el otro al momento de realizar la tarea comunitaria. En este sentido, estudios previos en temáticas similares contribuyeron de manera teórica y práctica y reconocieron la variedad de 
emociones desplegadas frente a tareas de lectura que se llevaron a cabo en clases de Lengua y Literatura (Martinenco et al., 2020).

La elección de la metodología de diseño estuvo ligada a la posibilidad que otorgan estos estudios para generar mejoras en los ambientes educativos a partir del diseño de una intervención; es decir, posibilita que los resultados puedan emplearse para evaluar e introducir mejoras en las prácticas de educación (Anderson y Shattuck, 2012).

En definitiva, el propósito de este trabajo se centra entonces en comprender las emociones desplegadas por un grupo de estudiantes de Educación Secundaria frente a tareas de escritura que realizaron en comunidad, considerando fundamentalmente la dinámica emocional y los modos de participación que se despliegan en este proceso.

\subsection{Participantes}

Según las especificaciones de la metodología de diseño, este estudio contó con la participación de tres agentes: estudiantes, docente e investigadora.

En primer lugar, participó la totalidad de estudiantes de sexto año de una institución privada de Educación Secundaria situada en una localidad de la provincia de Córdoba, Argentina. El grupo estuvo conformado por 39 estudiantes con una edad promedio de $\mathrm{M}=17,38$ años, quienes en 2019 cursaban su último curso de Educación Secundaria en el Ciclo Orientado en Ciencias Sociales.

En segundo lugar, participó la docente de Lengua y Literatura del mencionado sexto año. Es importante destacar que el rol de la docente es fundamental en los estudios con enfoque de diseño, ya que permite realizar acuerdos para crear conjuntamente con la investigadora un diseño instructivo e implementarlo en las clases.

En tercer lugar, tal como se mencionó en el párrafo previo, la investigadora participó en el estudio diseñando una secuencia de tareas académicas junto con la docente de Lengua y Literatura para que luego ella la implemente en las clases.

La selección de los participantes se efectuó en base a decisiones de la investigadora según las características de la investigación y la disponibilidad institucional.

\subsection{Estrategias de recolección de datos}

A los fines propuestos para este escrito se realizaron observaciones de clases, se analizaron tareas académicas y se llevaron a cabo entrevistas semiestructuradas a los estudiantes.

\section{Observaciones de clases}

A fin de conocer cómo se implementó el diseño que se creó conjuntamente con la docente de Lengua y Literatura en el contexto de aprendizaje estudiado, se efectuaron 16 observaciones de clases. Se observaron un total de 26 horas desde el día 5 de agosto de 2019 al 4 de noviembre del mismo año.

\section{Descripción de las tareas académicas}

Las tareas académicas que se diseñaron con la docente de Lengua y Literatura fueron pensadas como oportunidades prometedoras para el aprendizaje colaborativo y el despliegue de emociones positivas. El diseño mencionado incluyó el despliegue de dos grandes secuencias o temáticas: 1) literatura argentina contemporánea y 2) texto argumentativo. Dentro de cada tema se realizaron diferentes tareas académicas, la mayoría de las cuales fueron producciones escritas grupales. A continuación se hará referencia a algunas de estas actividades, las cuales fueron seleccionadas para ser comentadas a los fines de este escrito.

Tareas académicas desarrolladas en la secuencia de literatura argentina contemporánea. El primer tema, literatura argentina contemporánea, se abordó a partir de una novela de la escritora María Teresa Andruetto ${ }^{1}$. En esta secuencia se desarrollaron cuatro tareas académicas mediante las cuales se pretendió que los estudiantes conozcan y analicen una obra de la autora, con la intención final de crear una presentación visual

\footnotetext{
1 María Teresa Andruetto es una reconocida escritora argentina, nacida en 1954 en una localidad de la provincia de Córdoba. Su obra abarca mayormente literatura infantil y juvenil, aunque también incursiones en poesía y ensayo. Los ejes de sus escritos versan sobre la identidad, la dictadura argentina y lo femenino. Recibió numerosas distinciones, entre las cuales destaca el premio Hans Christian Andersen (2012), otorgado por la Organización Internacional para el Libro Juvenil. Fue la primera escritora argentina en recibir dicha mención.
} 
para la visita de la escritora a la institución. A continuación se describen las tareas que formaron parte de esta secuencia.

La primera tarea fue la lectura de la novela «La mujer en cuestión» ${ }^{2}$, escrita por la autora mencionada. Esta actividad se llevó a cabo en 6 clases de Lengua y Literatura, mientras la docente y algunos estudiantes leyeron en voz alta para el resto del grupo. Paralelamente, los jóvenes fueron realizando avances de lectura de modo individual en sus domicilios.

La segunda tarea consistió en leer tanto textos argumentativos y de resumen acerca de la novela abordada como entrevistas realizadas a su autora. Las lecturas se llevaron a cabo en pequeños grupos y luego los estudiantes hicieron una breve síntesis oral de la información que contenían los materiales.

La tercera tarea fue la creación grupal o individual de fotopoemas. Estas producciones, que debían inspirarse en algún aspecto de la novela «La mujer en cuestión», constituyeron parte del cierre de la secuencia y tuvieron carácter evaluativo. Los fotopoemas incluyeron imagen y escritura; en este sentido los estudiantes pudieron optar por redactar escrituras personales o extraer fragmentos de la novela o de otros textos; también se les permitió seleccionar imágenes de internet o tomar fotografías propias. Para ello emplearon celulares, computadoras y cámaras fotográficas. Cabe aclarar que fue necesario que los estudiantes avancen sus fotopoemas en horas extraescolares. La mayoría de estas producciones fueron realizadas en grupo, a excepción de unos pocos estudiantes que las crearon de modo individual.

La cuarta tarea estuvo dada por la visita de María Teresa Andruetto a la institución escolar. Específicamente el grupo incluido en este estudio armó un panel en las paredes exteriores de su aula con la totalidad de los fotopoemas realizados. También participaron en la realización de preguntas a la escritora durante una entrevista.

Tareas académicas desarrolladas en la secuencia de texto argumentativo. Luego de la secuencia vinculada a la literatura argentina contemporánea, el estudio continuó con el diseño instructivo acerca del segundo tema: texto argumentativo. Para abordar esta temática se desarrollaron tres tareas cuyo hilo conductor fueron las tecnologías en la vida cotidiana, con la intención de promover en los estudiantes la reflexión, argumentación y reconocimiento de posiciones acerca del tema para finalizar con una producción destinada a estudiantes del Ciclo Básico. A continuación, se especifican las tareas llevadas a cabo dentro de esta parte del diseño.

La primera tarea fue la visualización en el televisor del aula del capítulo "Caída en picada" de la serie Black Mirror, el cual refleja situaciones de un mundo ficticio en que las personas pueden puntuar y ser puntuadas a partir de cada interacción social. Posteriormente se realizó un debate y análisis oral del capítulo a partir de interrogantes realizados por la docente.

La segunda tarea constó de la lectura grupal o individual de dos textos que argumentan respectivamente a favor y en contra de la inclusión de las tecnologías en el cuento clásico Hansel y Gretel. Luego de la lectura, los estudiantes respondieron dos guías de trabajo; las mismas incluyeron consignas de dos tipos: un grupo apuntaba a respuestas que se obtenían directamente de los escritos mientras que otro grupo solicitaba respuestas que requerían elaboración y creación personal por parte de los estudiantes. Este es el caso, por ejemplo, de una consigna que indicaba a los estudiantes reescribir un cuento clásico en el que se incluya la tecnología en alguna situación.

La tercera tarea consistió en la producción grupal final de la secuencia: una infografía. Para ello, en primer lugar los participantes escribieron y administraron cuestionarios online a los destinatarios de la infografía -los estudiantes de cursos del Ciclo Básico-, a fin de comprender sus conocimientos acerca de problemáticas ocasionadas por la irrupción de las tecnologías: grooming, sexting, phishing, fake news, ciberbullying, entre otras. En segundo lugar, los estudiantes diagramaron y escribieron su infografía grupal. Para ello, seleccionaron los aspectos formales y de contenido de sus producciones empleando celulares y/o computadoras a fin de utilizar herramientas como Canva ${ }^{3}$, por ejemplo. La producción final fue enviada de modo online a los estudiantes del Ciclo Básico; además las infografías fueron impresas y mostradas a los destinatarios para, finalmente, quedar expuestas en las aulas del Ciclo Básico.

\section{Entrevistas a estudiantes}

Para recabar datos acerca de las apreciaciones de estos participantes sobre el diseño implementado en las clases se realizaron seis entrevistas grupales, por lo que se cuenta con las expresiones de doce estudiantes.

\footnotetext{
2 «La mujer en cuestión» es una novela de la escritora argentina María Teresa Andruetto, publicada en el año 2003 y ganadora del Premio Nacional de las Artes. El libro versa sobre la vida de Eva, una mujer que militó en la década del setenta y fue detenida durante la última dictadura militar argentina.

${ }^{3}$ Canva es un sitio web que contiene herramientas simples de diseño gráfico, las cuales permiten crear folletos, logos, calendarios, horarios, pósteres, flyers, invitaciones, diplomas, entre muchas otras posibilidades.
} 
Las entrevistas, de una duración aproximada de 25 minutos, fueron semiestructuradas y pretendieron indagar acerca de las actividades realizadas en las clases y las emociones vinculadas a ellas.

\subsection{Procedimientos}

El trabajo con los participantes inició en el mes de julio de 2019, cuando se comenzaron a diseñar las secuencias de clases conjuntamente con la docente de Lengua y Literatura.

Desde agosto a noviembre del mismo año se implementaron las tareas académicas previstas, las cuales fueron identificadas a partir de observaciones de clases.

A fines de noviembre de 2019 se llevaron a cabo las entrevistas en los horarios de clases de Lengua y Literatura. Previamente se explicitó a los participantes el carácter voluntario y confidencial de las mismas.

Los datos obtenidos se analizaron siguiendo los estudios de diseño, adoptando un énfasis cualitativo (Maxwell, 2019) y a partir de las categorías que iban emergiendo del contexto.

\section{Análisis y resultados}

Tal como puede vislumbrarse a partir de la descripción previa, algunas escrituras estuvieron más pautadas y dirigidas por la docente, mientras que en otras tareas los estudiantes pudieron escribir libremente; la mayoría de las producciones escritas fueron realizadas en pequeños grupos, aunque hubo unas pocas individuales y también existió variedad respecto a la inclusión de las tecnologías en las actividades.

En este proceso los estudiantes fueron experimentando diferentes emociones desplegadas de manera dinámica, a partir de la relación con los otros y con las actividades. A continuación, se analizarán sus emociones frente a las actividades de escritura comunitaria realizadas en la secuencia didáctica que se desarrolló en las clases.

\subsection{Emociones frente a escritura libre}

Las expresiones de los estudiantes durante las entrevistas refieren a un disfrute evidente por las producciones de escritura libre, por aquellas actividades del diseño en las que pudieron tomar decisiones y ser autónomos en variados aspectos.

Dentro de este grupo de tareas encontramos, por ejemplo, la creación de un fotopoema, la redacción de una encuesta online, la reescritura de un cuento clásico que incluye tecnologías y/o la diagramación y escritura de una infografía. En estas y otras actividades que requerían escrituras de características similares los estudiantes partían de una consigna inicial que indicaba qué tipo de escrito realizar, pero podían elegir tanto el contenido como la estética de sus producciones; la docente les brindó libertad para expresar sus sentimientos y opiniones, elegir qué información incluir, seleccionar la combinación de aspectos formales y de contenido, entre otros. La imaginación, la creación y la identificación con su escritura fueron destacadas en las entrevistas como aspectos positivos de la escritura libre. Se presentan a continuación algunos fragmentos:

Estudiante 3: No me aburrió la actividad porque requería mucha opinión personal y escritura propia, entonces eso sí me gustó.

Estudiante 5: (Para el fotopoema) Fue pensar en algo, en alguien y el factor imaginación para escribir. Eso fue re lindo porque dejás fluir la imaginación. Después la foto estuvo buena porque la tenés que editar y también es divertido. Estuvo bueno.

Estudiante 6: A mí me gustó porque no me costó, me salió así. Yo leí el libro y después pensé un microrrelato poniéndome en el lugar de la protagonista, entonces desde ahí me salió el escrito.

Esta manera de escribir sin dar respuesta a interrogantes específicos sino expresándose más libremente fue percibida por los estudiantes como una tarea rápida, fácil y divertida. Se trata de escrituras que les permitieron autorregular sus aprendizajes ya sea de manera individual o grupal -según el modo en que llevaron a cabo las producciones-, aspecto que incidiría positivamente en las emociones de los estudiantes (Pekrun, 2006), quienes lograron un disfrute y un gusto por la tarea.

Para este grupo de estudiantes serían importantes tanto la autonomía como el control al momento de conducir su propio proceso de aprendizaje, por lo que valoran las consignas que les permiten realizar escrituras en las que pueden elegir la modalidad de presentación, la organización del tiempo y de los grupos de trabajo, el contenido, los aspectos formales, entre otros aspectos. Es interesante destacar que estas expresiones de los participantes condicen con los resultados obtenidos a partir de las observaciones grupales, ya que en estas tareas los estudiantes se mostraron entusiasmados, participativos, comunicativos y contentos. 
A partir de lo interpretado previamente, pudimos analizar cómo se generaron condiciones y consignas para que los estudiantes desplieguen y co-construyan su autonomía. En términos de la teoría retomada, se considera importante continuar proponiendo este tipo de tareas de aprendizaje en las clases, que posibiliten a los estudiantes controlar y autorregular sus escrituras, puesto que un alto nivel de control subjetivo otorgaría más posibilidades de que los estudiantes desplieguen emociones positivas (Pekrun, 2006).

\subsection{Emociones frente a escritura grupal}

La descripción de las tareas realizada previamente permitió vislumbrar que la amplia mayoría de las actividades de escritura llevadas a cabo en el diseño instructivo fueron grupales.

La realización de respuestas luego de leer un texto, la recreación de un cuento clásico en que se introducen las tecnologías como parte del argumento o la realización de una infografía son ejemplos de producciones donde los participantes escribieron junto con sus compañeros. De acuerdo a las entrevistas analizadas, las emociones preponderantes en este tipo de tareas fueron disfrute y alegría, dado que desempeñaron un trabajo conjunto que brinda seguridad a los integrantes del equipo y le permite a cada uno adquirir cierto grado de responsabilidad y compromiso. A continuación, algunos fragmentos ejemplifican lo expuesto:

Estudiante 1: Yo lo re disfruto porque es con mis amigas entonces, mientras hacemos la actividad, también te reís, sacás otro tema y así es más llevadero.

Estudiante 2: La escritura grupal ayuda; vos decís a lo mejor “esto para mí está bien” y la otra "ah, sí, para mí también” y ahí como mejor, (me siento) más segura.

Estudiante 3: (Elijo escribir en grupo) más que nada por la responsabilidad y el compromiso que tiene cada una.

En el sentido de este último fragmento, Bar-On (2012 en Paoloni, 2019) menciona a la responsabilidad social como un factor del componente interpersonal, la cual incluye habilidades para participar en relaciones sociales de manera constructiva y cooperativa. Este trabajo conjunto de los estudiantes a partir de la comprensión del rol del otro y la asunción del propio, muestra ciertas características de la inteligencia interpersonal propuesta por Gardner (2016).

Además, muchos estudiantes expresan su satisfacción al momento de realizar escrituras grupales por los beneficios que ello trae para la producción final; según sus expresiones, se obtendrían escritos integrales y creativos, los cuales complementan e incluyen las perspectivas de todos los participantes:

Estudiante 9: Obviamente me gusta trabajar en grupo porque cada uno tiene ideas que yo no tengo, piensa algo que yo no pienso, entonces ahí vamos complementando y hay cosas mucho más creativas.

Estudiante 10: Prefiero hacerlo en grupo por la razón de que debatimos, (...) entonces es como que de los dos siempre sale algo mejor de lo que me puede salir a mí. (...) Aprendés a escuchar al otro, a valorar lo que dice el otro y aprendés bastante en la redacción.

Estudiante 11: A lo mejor yo escribo de una forma y a lo mejor el otro, de otra y complementamos nuestras ideas. (...) Juntás varias posturas, varios conocimientos, varios datos, varias formas de pensar y los incluís en un solo texto,

A partir de las expresiones de los estudiantes es posible comprender el alto valor que otorgan a la escritura grupal como modo de complementar ideas, perspectivas y posicionamientos que ampliarían el conocimiento y redundaría en un trabajo de mayor riqueza. Es interesante reconocer que el trabajo conjunto es un proceso que culmina en un resultado compartido, el cual es consecuencia de acuerdos, negociaciones y emociones que fluyen en el transcurso de ello. Este tipo de habilidades tienen que ver con la conciencia social y la posibilidad de considerar una perspectiva diferente a la propia, a partir de la empatía tanto cognitiva como emocional (Weissberg et al., 2015).

Por otra parte, tal como se plasma en los fragmentos, no es menor que los participantes destacan un aprendizaje social -más allá del cognitivo-, vinculado a la división de tareas, la escucha de opiniones ajenas y la valoración de lo que el otro puede aportar a la escritura grupal. Estas habilidades junto a otras como la comunicación verbal y no verbal, los comportamientos cooperativos, la escucha activa, la negociación frente a los conflictos y la solicitud de ayuda tienen que ver con destrezas que son necesarias para llevar a cabo relaciones sociales gratificantes y saludables (Weissberg et al., 2015).

A partir de las expresiones que los participantes tuvieron en las entrevistas, podemos vislumbrar la preferencia de este grupo de estudiantes por la escritura grupal. En este proceso se ponen en juego diversas habilidades socioemocionales, las cuales fueron describiéndose previamente, dentro de las que se destaca el establecimiento de relaciones satisfactorias con los compañeros y la participación con una actitud cooperativa y constructiva. 
En este apartado interesó destacar la importancia que asumen las emociones y las habilidades socioemocionales en el trabajo grupal, al incidir en el bienestar académico de los estudiantes (Paoloni, 2019).

\subsection{Emociones frente a escritura creativa con Tecnologías de la Información y la Comunicación (TIC)}

A lo largo del desarrollo de la secuencia didáctica, la mayoría de las actividades realizadas incluyeron fundamentalmente el uso del teléfono móvil, aunque también se emplearon asiduamente las computadoras personales y la televisión del aula. Las tecnologías fueron empleadas en todo tipo de tareas, tales como crear un fotopoema en Canva partiendo de una fotografía tomada por los estudiantes con sus teléfonos o cámaras digitales, la redacción de entrevistas en formularios de Google, la reescritura de un cuento clásico que luego se subió a un padlet colaborativo online, el visionado de un capítulo de una serie de Netflix, la lectura de consignas desde la televisión, entre otras.

Si se consideran específicamente las actividades de escritura con TIC, en las entrevistas los estudiantes refieren fundamentalmente a su experiencia durante la creación del fotopoema y la infografía, es decir, a aquellas tareas en las que se brindó oportunidad de realizar escrituras creativas y libres. A continuación brindamos algunos ejemplos de las percepciones de los participantes acerca del empleo de las tecnologías en este tipo de producciones:

Estudiante 3: Muy bueno, para mí, muy bueno porque dibujar no se me da entonces me es más fácil. Aparte que me lleva menos tiempo y en internet tengo la posibilidad de ver muchas imágenes y si quiero esta, la pego y voy complementando; y si a los dos minutos veo otra, la cambio; es posible la edición.

Estudiante 5: Es rápido, es fácil y es divertido porque tenés aplicaciones para hacerlo. Podés cambiar la letra, el color, todo.

Estudiante 10: Yo creo que es más entretenido. Yo siempre prefiero estudiar desde el teléfono o hacer algo desde el teléfono que a mano, porque no soy prolija y me resulta más entretenido porque podés poner música mientras lo hacés o..., no sé, te despeja un poco más.

Tal como se vislumbra en los fragmentos anteriores, los estudiantes experimentan emociones vinculadas al disfrute, el entusiasmo y la satisfacción al emplear las tecnologías para realizar estas escrituras fundamentalmente porque les resultan herramientas que otorgan beneficios como la posibilidad de edición, prolijidad, entretenimiento, sencillez, ahorro de tiempo, entre otros. En este sentido, Prensky (2001 y 2004 en Cassany, 2012) refiere a esta preferencia de los estudiantes por la multitarea -realizar una escritura y escuchar música al mismo tiempo-, la multimodalidad -incorporar texto, imágenes, audio en una misma producción- y el aprendizaje que se construye de manera divertida.

Hand y Prain (2012) destacan la importancia de la multimodalidad al construir textos científicos en ciencias y la necesidad de escribir para audiencias reales. En este sentido, se considera interesante que también en el área de Lengua y Literatura los estudiantes tengan la oportunidad de redactar textos multimodales como el fotopoema o la infografía, por ejemplo, destinados a un público real -los estudiantes de Ciclo Básico, en el caso de las infografías- y a situaciones concretas -la visita de María Teresa Andruetto, si consideramos el fotopoema-.

Podríamos pensar que los estudiantes experimentan un sentir positivo al asumir el rol de autores de sus escrituras creativas, tal como hacen con sus redes sociales. Disfrutan crear producciones multimodales y libres, tomando inspiración y recursos de la web. Esto es central y va en consonancia con lo que propone Cassany (2012), ya que la principal diferencia que el autor encuentra al escribir con tecnologías reside justamente en hacerlo de manera online, en línea, en conexión con una multitud de recursos provenientes de diferentes partes del planeta. Podemos pensar que no existirían grandes diferencias al hacer una infografía de manera manual que si la hacemos en una computadora sin conexión de internet; la diferencia está en la conectividad que posibilitó a los estudiantes acceder a recursos como traductores, diccionarios, plantillas, entre otros. Este tipo de escritura en línea se caracteriza por contar con más cantidad de interlocutores y documentos, por promover la cooperación, el aprendizaje compartido y la diversificación de la escritura, ya que actualmente se difunden muchas formas escritas no normativas -los estudiantes escribieron sus producciones con fuentes poco convencionales, con predominio de puntos suspensivos, redactando textos cortos y directos y empleando masivamente los ítems-.

En definitiva, encontramos que el empleo de tecnologías para este tipo de escrituras habilitó nuevas formas de producción de conocimiento al promover el trabajo colaborativo, la polifonía de voces y la construcción colectiva (Maggio et al., 2014). 


\section{Discusión y conclusiones}

El estudio desarrollado con los estudiantes de sexto año permitió comprender la dinámica emocional desplegada frente a tareas de escritura comunitaria y los modos de participación de los participantes. Tomando en consideración la secuencia de actividades incluidas en ambas temáticas -literatura argentina contemporánea y texto argumentativo- es posible reconocer el despliegue de emociones de todo tipo. Los resultados alcanzados denotan el surgimiento de un sentir positivo cuando este grupo de estudiantes realiza escrituras con otros, donde es central el establecimiento de acuerdos y el reconocimiento de las motivaciones, intenciones y estados de ánimos de los demás (Gardner, 2016).

Las emociones positivas también fueron desplegadas frente a escrituras que posicionaron a los estudiantes como autores y protagonistas, en las cuales lograron decidir aspectos formales y de contenido según el objetivo de la tarea y sus destinatarios. De esta manera, los participantes se involucraron en prácticas de escritura no sólo por una necesidad escolar sino también social y cultural; este es el caso de la creación de la infografía para los estudiantes del Ciclo Básico o la realización del fotopoema a modo de bienvenida a la escritora María Teresa Andruetto.

Se advierte una marcada preferencia por escrituras multimodales que puedan ser organizadas por los mismos estudiantes, destacando así el disfrute por la autorregulación de sus aprendizajes. En este sentido, Pekrun (2006) postula que la autonomía y el control por los aprendizajes propios incide en la posibilidad de experimentar emociones académicas positivas.

Asimismo, interesa destacar el hilo conector acerca del tema implicado en la secuencia de argumentación, ya que todas las tareas -no sólo las de escritura- se vincularon al tópico del empleo de las tecnologías en la actualidad. Resulta interesante, en términos de Pérez-Abril (2004), que las tareas académicas se liguen a intereses y expectativas de los implicados.

Los hallazgos permiten entender cómo ciertas emociones acercaron más a los estudiantes al bienestar mientras que otras lo hicieron en menor medida. Aquí Mellado et al. (2014) diferencian ciertas emociones positivas -alegría, orgullo y gratitud-, emociones negativas -miedo, ira, culpa- y emociones neutras, como la sorpresa. Es de destacar, tal como sucede en un estudio en Ciencias realizado por Dávila Acedo et al. (2014), que los dos tipos de emociones coexisten al aprender. Sin embargo acordamos con Bisquerra (2016a) al advertir que esto no implica necesariamente que las emociones positivas sean buenas y las emociones negativas, malas; es preciso no confundir la valencia de las emociones con su valor. Por el contrario, la dinámica emocional es necesaria y esperable en los procesos de aprendizaje por los que transitamos.

En suma, se considera que el aporte del estudio radica, tal como lo indican la metodología de diseño, en aspectos prácticos o pedagógicos y en aspectos teóricos.

El aporte práctico se refiere a la utilidad para pensar el diseño de tareas de escritura con ciertas características -libres, comunitarias, mediadas por tecnologías- a fin de promover emociones positivas. De modo similar a lo que proponen Mellado et al (2014), aunque en el área de Ciencias, el desafío consiste en promover actividades creativas, emocionantes y placenteras que promuevan orgullo, alegría y satisfacción. Sin embargo, en este punto no es posible dejar de hacer dos aclaraciones; en primer lugar, se conoce la importancia de realizar diferentes tipos de escrituras y no sólo aquellas que presentan las mencionadas características, dado que es preciso que los estudiantes se enfrenten con multiplicidad de tareas, textos, desafíos y emociones. En segundo lugar, se destaca que las diferentes tareas de aprendizaje promueven emociones dinámicas y que fluctúan en las personas puesto que las emociones involucran experiencias subjetivas en los estudiantes, de modo que es posible experimentar diferentes emociones frente a una misma tarea (Pekrun, 2014). En definitiva, de acuerdo con Dávila Acedo et al. (2016) este tipo de estudios brinda la posibilidad de mejorar las situaciones de enseñanza con la intención de promover emociones positivas, despertando el interés de los estudiantes y articulando el trabajo individual y colaborativo.

El aporte teórico del estudio estriba en un aumento del conocimiento en el campo acerca de la importancia que adquieren los aspectos emocionales y sociales de los estudiantes, ya sea para aumentar beneficios a nivel personal como a nivel social (Fernández-Berrocal et al., 2017).

Por el contrario, las limitaciones de la investigación están dadas por la dificultad para permanecer más tiempo en el contexto, lo cual permitiría analizar la conformación -o no- de una comunidad de aprendizaje. Además, considerando la complejidad de los contextos, sería necesario considerar en los estudios otros aspectos de los aprendizajes.

Se encuentra relevante para investigaciones futuras incorporar otras herramientas de recolección de datos, tales como grupos de discusión, narrativas, entre otros. Además, es importante continuar empleando metodologías de diseño en estudios del área educativa y, específicamente, vinculados a las tecnologías puesto que es innegable la incidencia de las mismas en nuestra sociedad, de modo que resultaría interesante analizar el efecto del uso de tecnologías en contextos de aprendizaje formales, no formales e informales (Esteve- 
Mon et al., 2019). Al considerar estos diferentes ámbitos, resulta de interés también realizar estudios acerca de la hibridación de contextos y el modo en que van conformando ecologías de aprendizaje.

\section{Apoyos}

La investigación en que se enmarca el presente estudio se encuentra financiada por una Beca Doctoral otorgada por el Consejo Nacional de Investigaciones Científicas y Técnicas (CONICET) según resolución nº 1456/2018.

\section{Referencias}

Anderson, T. y Shattuck, J. (2012). Design-based research: A decade of progress in education research? Educational Researcher, 41(1), 16-25. DOI: 10.3102/0013189X11428813

Bisquerra, R. (2016a) 10 ideas clave. Educación emocional. GRAÓ.

Bisquerra, R. (2016b). Universo de emociones: la elaboración de un material didáctico. En J. L. Soler, L. Aparici, O. Díaz, E. Escolano y A. Rodríguez (Coords.), Inteligencia emocional y Bienestar II. Reflexiones, experiencias profesionales e investigaciones (pp. 20-31). Ediciones Universidad San Jorge. https://bit.ly/3iLkTMo

Bisquerra, R. (2018). Educación emocional para el desarrollo integral en secundaria. Aula de secundaria, 28, 10-15. https://bit.ly/2FJRwLO

Cassany, D. (2012). En_linea: leery escribir en la red. Anagrama.

Chóliz, M. (2005). Psicología de la emoción: El proceso emocional. https://bit.ly/3iz8dYV

Dávila Acedo, M. A., Borrachero Cortés, A. B., Brígido Mero, M. y Costillo Borrego, E. (2014). Las emociones y sus causas en el aprendizaje de la física y la química. Revista INFAD de Psicología. International Journal of Developmental and Educational Psychology, 4(1), 287-294. DOI: 10.17060/ijodaep.2014.n1.v4.614

Dávila Acedo, M. A., Cañada Cañada, F. Sánchez Martín, J. y Mellado Jiménez, V. (2016). Las emociones en el aprendizaje de física y química en educación secundaria. Causas relacionadas con el estudiante. Educación química, 27(3), 217-225. DOI: 10.1016/j.eq.2016.04.001

Díez-Palomar, J. y Flecha-García, R. (2010). Comunidades de Aprendizaje: un proyecto de transformación social y educativa. Revista Interuniversitaria de Formación del Profesorado, 24(1), 19-30. https://bit.ly/32xQtI5

Esteve-Mon, F., Cela-Ranilla, J. M. y de Benito-Crosetti, B. (2019). DBR: una estrategia metodológica para investigar en tecnología educativa. En M. G. Cervera, V. E. González y J. L. L. Cantabrana (Eds.), ¿Cómo abordar la educación del futuro?: Conceptualización, desarrollo y evaluación desde la competencia digital docente (1 $1^{a}$ edición, pp. 65-76). Octaedro.

Fernández-Berrocal, P., Cabello, R. y Gutiérrez-Cobo, M. J. (2017). Avances en la investigación sobre competencias emocionales en educación. Revista interuniversitaria de formación del profesorado, 88, 15-26. https://bit.ly/2FEA8s8

Gardner, H (2016). Estructuras de la mente: La teoría de las inteligencias múltiples. Fondo de cultura económica.

Gobierno de Córdoba. Ministerio de Educación. Secretaría de Educación. Subsecretaría de Promoción de Igualdad y Calidad Educativa. (2018). Tomo 4. Oportunidades curriculares para el aprendizaje emocionaly social en Educación Secundaria. https://bit.ly/3hthVec

Goetz, T., Pekrun, R., Hall, N. y Haag, L. (2006). Academic emotions from a social-cognitive perspective: Antecedents and domain specificity of students' affect in the context of Latin instruction. British Journal of Educational Psychology, 76(2), 289-308. DOI: 10.1348/000709905X42860

González, A., Paoloni, V.,y Rinaudo, M. C. (2013). Boredom and enjoyment in the Spanish language classroom in secondary education: Motivational predictors and effects on performance. Anales De Psicologia / Annals of Psychology, 29(2), 426-434. DOI:10.6018/analesps.29.2.136401

Gutiérrez, K. D. y Penuel, W. R. (2014). Relevance to practice as criterion for rigor. Educational Researcher, 43(1), 19-23. DOI: 10.3102/0013189X13520289

Hand, B. y Prain, V. (2012). Writing as a learning tool in science: lessons learnt and future agendas. En B. J. Fraser, K. Tobin y C. J. McRobbie (Eds.), Second international handbook of science education (pp. 1375-1384). Springer Netherlands. DOI: 10.1007/978-1-4020-9041-7_88

Järvenoja, H. y Järvelä, S. (2009). Emotion control in collaborative learning situations: Do students regulate emotions evoked by social challenges. British Journal of Educational Psychology, 79(3), 463-481. DOI: 10.1348/000709909X402811

Maggio, M., Lion, C. y Perosi, M. (2014). Las prácticas de la enseñanza recreadas en los escenarios de alta disposición tecnológica. Polifonías Revista de Educación, 3(5), 101-127. https://bit.ly/32xL5EM 
Martín, R. B. (2019). Perspectiva teórica sobre el estudio de las comunidades de aprendizaje en R. B. Martín, M. C. Rinaudo y P. V. Paoloni (Comps.), Comunidades. Estudios y experiencias sobre contextos y comunidades de aprendizaje (pp. 75-131). Eduvim.

Martín, R. B., Vaja, A. B. y Paoloni, P. V. (2015). Emociones y participación en contextos no formales. Los casos de un taller de tejido y un curso de socorristas. Profesorado: Revista de Curriculum y Formación de Profesorado, 19(2), 185-202. https://bit.ly/32vqDV8

Martinenco, R. M., Vaja, A. B. y Martín, R. B. (2020). Emociones académicas en una secuencia didáctica de Lengua y Literatura en Educación Secundaria. Quaderns de Psicología, 22(1), e1495. DOI: 10.5565/rev/qpsicologia.1495

Maxwell, J. (2019). Diseño de investigación cualitativa. Gedisa.

Mellado, V. Borrachero, A. B., Brígido, M., Melo, L., Dávila, M. A., Cañada, F., Conde, M. C., Costillo, E., Cubero, J., Esteban, R., Martínez, G., Ruiz, C. Sánchez, J. Garritz, A., Mellado, L., Vázquez-Bernal, Jiménez, R. y Bermejo, L. (2014). Las emociones en la enseñanza de las ciencias. Enseñanza de las Ciencias, 32(3), 11-36. DOI: $10.5565 / \mathrm{rev} /$ ensciencias.1478

Ochoa de Alda, J. A. G., Marcos-Merino, J. M., Méndez-Gómez, F. J., Mellado-Jiménez, V. y EstebanGallego, M. R. (2019). Emociones académicas y aprendizaje de biología, una asociación duradera. Enseñanza de las ciencias: revista de investigación y experiencias didácticas, 37(2), 43-61. DOI: $10.5565 / \mathrm{rev} /$ ensciencias. 2598

Paoloni, P. (2019). Competencias socioemocionales antes, ahora... ¿y mañana? En P. V. Paoloni, M. C. Rinaudo y R. B. Martín (Comps.), Yo, tú... Ellos y nosotros. Competencias socioemocionales en la construcción de identidades profesionales ( $1^{\circ}$ edición, pp. 103-134). Brujas.

Pekrun, R. (2006). The control-value theory of achievement emotions: Assumptions, corollaries, and implications for educational research and practice. Educational Psychology Review, 18(4), 315-341. DOI: $10.1007 / \mathrm{s} 10648-006-9029-9$

Pekrun, R. (2014). Emotions and learning. Educational practices series, 24, 2-31. https://bit.ly/2H3Bhds

Pérez-Abril, M. (2004). Leer, escribir, participar: un reto para la escuela, una condición de la política. Lenguaje, 32, 71-88. DOI: 10.25100/lenguaje.v32i0.4813

Putwain, D. W., Schmitz, E. A., Wood, P. y Pekrun, R. (2020). The role of achievement emotions in primary school mathematics: Control-value antecedents and achievement outcomes. British Journal of Educational Psychology. DOI: $10.1111 /$ bjep.12367

Reeve, J. (2010). Motivación y emoción (5 edición). McGraw Hill. https://bit.ly/32B3aC0

Reinmann, G. y Fiedler, S. H. D. (2017). Design-based research on the way to mainstream research? Comments on the plea for phronesis by Bardone and Bauters. EDeR - Educational Design Research, 1(1), 1-5. DOI: $10.15460 /$ eder.1.1.1050

Rinaudo, M. C. y Donolo, D. (2010). Estudios de diseño. Una perspectiva promisoria en la investigación educativa. RED - Revista de Educación a Distancia, 22. https://bit.ly/32xR6RX

Rodrígues de Mello, R. (2011). Comunidades de aprendizaje: democratización de los centros educativos. Tendencias pedagógicas, 17(7), 3-18. https://bit.ly/2H3BmxM

Vázquez-Bernal, B., de las Heras Pérez, M. Á. y Jiménez-Pérez, R. (2020). Identidad patrimonial, emociones y enseñanza de las Ciencias Experimentales. Didáctica de las Ciencias Experimentales y Sociales, 38, 153-170. DOI: $10.7203 /$ dces.38.15688

Weissberg, R. P., Durlak, J. A., Domitrovich, C. E. y Gullotta, T. P. (2015). Social and emotional learning: Past, present, and future. En J. A. Durlak, C. E. Domitrovich, R. P. Weissberg,y T. P. Gullotta (Eds.), Handbook of social and emotional learning: Research and practice (pp. 3-19). The Guilford Press. https://bit.ly/3hAp0K7

Wenger, E. (2010). Communities of practice and social learning systems: the career of a concept. En C. Blackmore (Ed.), Social Learning System and communities of practice (pp. 179-198). Springer London. DOI: 10.1007/978-1-84996-133-2_11 